\title{
Dynamo action and magnetic activity during the pre-main sequence : Influence of rotation and structural changes
}

\author{
Constance Emeriau-Viard \& Allan Sacha Brun \\ Laboratoire AIM Paris-Saclay, \\ CEA/DSM - CNRS - Université Paris Diderot, \\ IRFU/SAp Centre de Saclay, \\ F-91191 Gif-sur-Yvette Cedex, France \\ email: constance.emeriau@cea.fr \\ email: sacha.brun@cea.fr
}

\begin{abstract}
During the PMS, structure and rotation rate of stars evolve significantly. We wish to assess the consequences of these drastic changes on stellar dynamo, internal magnetic field topology and activity level by mean of HPC simulations with the ASH code. To answer this question, we develop 3D MHD simulations that represent specific stages of stellar evolution along the PMS. We choose five different models characterized by the radius of their radiative zone following an evolutionary track, from $1 \mathrm{Myr}$ to $50 \mathrm{Myr}$, computed by a 1D stellar evolution code. We introduce a seed magnetic field in the youngest model and then we spread it through all simulations. First of all, we study the consequences that the increase of rotation rate and the change of geometry of the convective zone have on the dynamo field that exists in the convective envelop. The magnetic energy increases, the topology of the magnetic field becomes more complex and the axisymmetric magnetic field becomes less predominant as the star ages. The computation of the fully convective MHD model shows that a strong dynamo develops with a ratio of magnetic to kinetic energy reaching equipartition and even super-equipartition states in the faster rotating cases. Magnetic fields resulting from our MHD simulations possess a mixed poloidal-toroidal topology with no obvious dominant component. We also study the relaxation of the vestige dynamo magnetic field within the radiative core and found that it satisfies stability criteria. Hence it does not experience a global reconfiguration and instead slowly relaxes by retaining its mixed poloidal-toroidal topology.
\end{abstract}

Keywords. Convection,Dynamo,Magnetohydrodynamics,Stars interiors

\section{Introduction}

During the pre-main sequence phase (PMS), stellar structure and rotation rate change drastically. As the star contracts, the rotation rate also evolves. It was modeled by MacGregor \& Brenner (1991) and Gallet \& Bouvier (2013) by three different phases : at the very beginning of the PMS the rotation rate remains constant as the star is still locked with its protostellar disk, then it increases as the radius of the star decreases, finally the star reaches a stable outer radius and the rotation rate decreases because of the wind-braking Réville (2015) The internal structure also significantly changes. At the very beginning of the PMS, stars are fully convective as there are no long-term thermo-nuclear reactions in their interior. As the star ages, hydrogen burning develops, the opacity of the core decreases and a stable radiative core appears and grows. These major changes impact the star's properties, especially their internal rotation and magnetic field. 
Stellar rotation rate and magnetic field are strongly linked through a feedback loop created by complex physical processes. Indeed stellar magnetic field impacts the transport of angular momentum (Brun et al. 2004; Strugarek 2017) trough the Maxwell stresses and also the braking of the star by the wind while stellar rotation influences the magnetic field through dynamo process, and especially through $\Omega$ effect. This mutual influence was shown observationally for instance by Pizzolato (2003), showing a correlation between coronal X-ray emission and stellar rotation rate at moderate and high Rossby number $R_{o}=P_{\text {ro }} / \tau_{\text {conv }}$ and also that either the surface field or the stellar dynamo, or both, saturates for fast rotation rates. Stellar internal structure also impacts the magnetic field topology and amplitude as shown by Gregory et al. (2012) (see also these proceedings). This study show that stars with a massive radiative core, $M_{\text {core }}>0.4 M_{*}$, possess a complex magnetic field, i.e. non axisymmetric and with weak dipole components. Stars with smaller radiative core, $0<M_{\text {core }}<0.4 M_{*}$, have a less complex and more axisymmetric field. In fully convective stars the behavior of stellar magnetic field might even be bistable with a mixture of different geometries and amplitudes (Morin 2010).

As the stellar radiative core increases as stars age along the PMS, we also wish to know how the magnetic field evolves in the radiative zone as convective dynamo action does not support it anymore. These magnetic fields are observed in massive stars, since their envelop is radiative, where they are often oblique dipole. These fields are highly unstable and Tayler (1973) and Markey \& Tayler (1973) showed that only a mixed poloidaltoroidal topology can be stable in a stably stratified radiative zone. Braithwaite (2008) introduced a stability criteria for a stable fossil field in a radiative zone $E_{\text {pol }} / E_{\text {tot }}<0.8$. It is interesting to assess if the magnetic field left over by the dynamo process is stable or if it must relax to a different configuration.

\section{Model setup}

To analyze the evolution of magnetic field during the PMS, we choose to compute 3D global magnetohydrodynamical models of a one solar mass star. However stellar evolution in the PMS lasts for several Myr whereas 3D MHD simulations can only compute, with reasonable resources, stellar evolution for several hundreds of years. Hence, we select specific models that represent the most important stages of the PMS. To characterize these models, we need quantitative values for luminosity, rotation rate, radii ... These physical values are given by stellar evolution models that were computed by the 1D stellar evolution code STAREVOL Amard (2016) as shown in Figure 1. We choose to select five models such that the radiative core radii (in stellar units) are well distributed, almost every $20 \%$, and the ratio between the rotation rate of two consecutive models is smaller than two (see Figure 1). Radial structures provided by STAREVOL simulations enable us to create reference states and thus initialize our 3D ASH simulations. Hence we compute hydrodynamical simulations for each of the fives models. We run the calculations until the hydrodynamical simulations have equilibrated internal flows and coupling between radiative core and the convective envelop.

To study the evolution of the stellar magnetic field through the PMS, we first injected a seed magnetic field in the fully convective hydrodynamical model. This weak seed confined dipole represents the field left by the proto-stellar phase. We run the magnetohydrodynamical simulation of the fully convective model until it reaches an equilibrium state with a dynamo generated field. Then we introduced the magnetic field resulting from this simulation into the $20 \%$ hydrodynamical simulation. Hence we can see how the change of internal structure affects the magnetic field. Once this simulation has reached an equilibrium state, in the statistically stationary sense, and the magnetic field has 


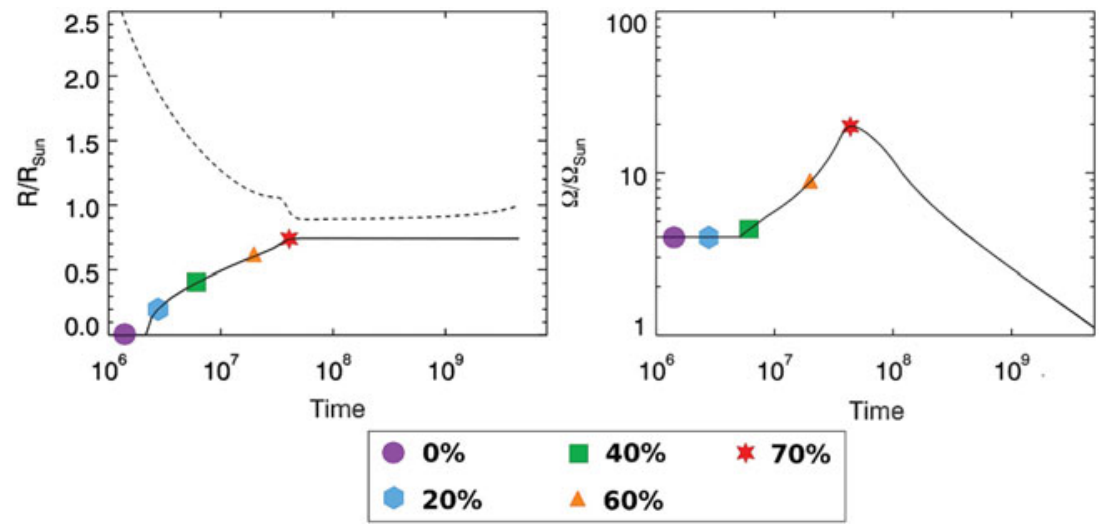

Figure 1. Choice of the 3D ASH models based on stellar evolution STAREVOL code. Left : Evolution of stellar radius (solid) and radius of the radiative core (dash) during the PMS and the MS. Right : Stellar rotation rate as a function of time. At the beginning of the PMS it is constant since star is still in the disk- locking phase. Then it increases as the star contracts under the effect of gravity until the ZAMS. Stellar rotation rate starts decreasing as the stellar contraction stops and magnetic wind brakes the star.

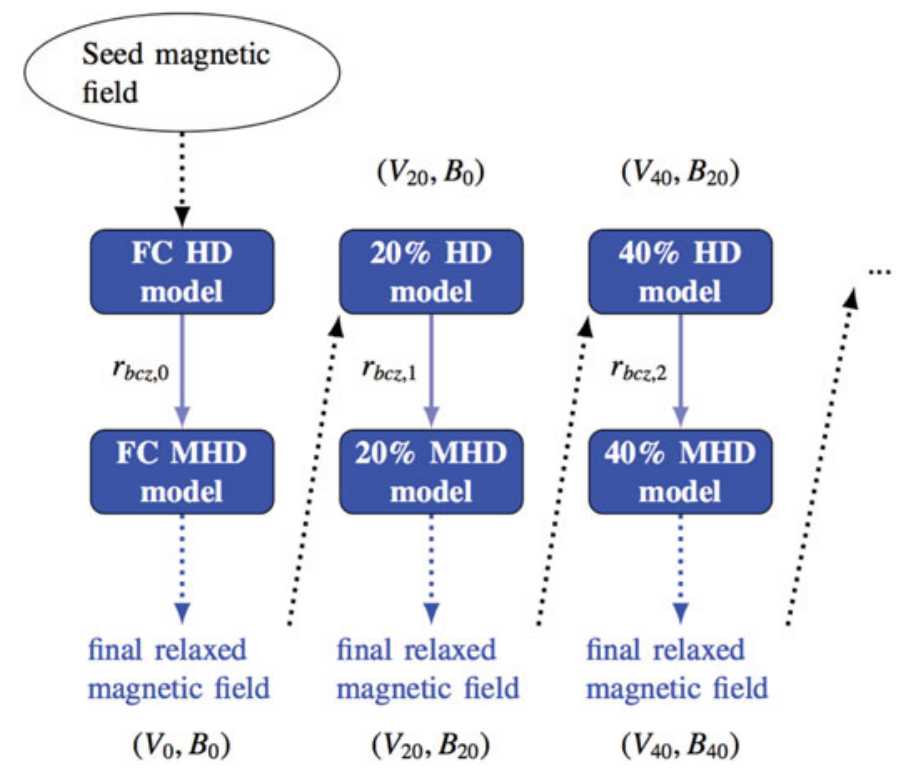

Figure 2. Description of the procedure to study the evolution of a stellar magnetic field through the PMS. In each HD simulation, we introduced the magnetic field resulting from the previous MHD simulation. MHD simulations are computed until they are equilibrated with dynamo process in the convective zone and a relaxation of the magnetic field in the radiative zone, if present. In the first model, i.e. the fully convective one, we choose to inject a weak seed confined dipole representing the field left by the proto-stellar phase.

relaxed in the radiative core, we introduce the resulting magnetic field in the following hydrodynamical model. By reproducing these operations with all HD models, we can analyze the influence on the magnetic field of the changes of internal structure and rotation rate caused by stellar evolution (see details in Emeriau-Viard \& Brun 2017). 


\section{HD}
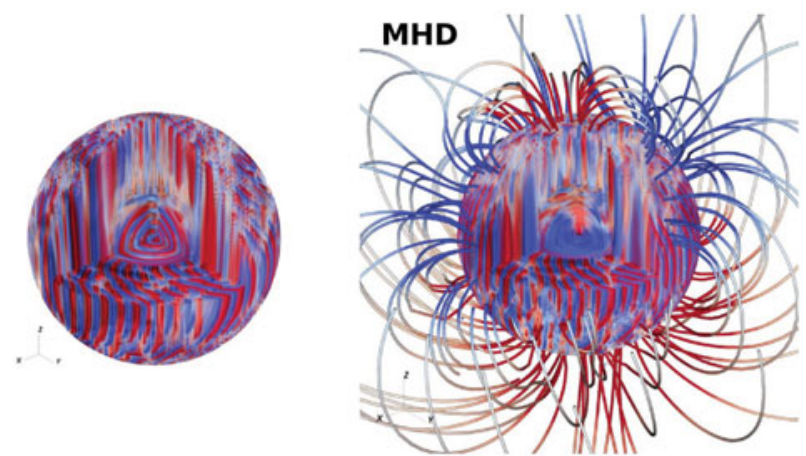

Figure 3. 3D views of the $40 \%$ model. Left: Radial velocity of the hydrodynamical model. Right: Radial velocity of the MHD model with the potential extrapolation of magnetic field outside the star. Upflows in red and downflows in blue. Field lines are color coded with the radial component of the magnetic field.

\section{Magnetic field properties and evolution during the PMS phase}

The introduction of magnetic field in the HD simulations strongly impacts the internal flows. Differential rotation is flatten due to the presence of Maxwell stresses. Hence the radial extent of the convective motions in the MHD models is larger than in the HD ones, since there is less horizontal shear. The quenching of differential rotation also have an influence on transport of angular momentum. Indeed viscous diffusion becomes negligible for the transport of angular momentum. Since Reynolds stresses is outward, as in the HD models, the inward transport is carried by Maxwell stresses. The 3D topology of radial velocity is illustrated for the $40 \%$ model in Figure 3 both for the hydrodynamical and magnetohydrodynamical cases. We notice the cylindrical patterns of convection linked to fast rotation in both cases. In Figure 3, we also see an extrapolation of the magnetic field outside the star. It shows us a potential extrapolation of the magnetic field outside the star which is complex, highly non-axisymmetric and exhibits as well extended transequatorial loops.

As the star evolves along the PMS, we want to study the evolution of the magnetic field both in amplitude and topology. Thus we define the dipole field strength as done by Christensen \& Aubert (2006). $f_{\text {dip }}$ is time-average ratio at the surface of the mean dipole field strength to the field strength in harmonic degrees $\ell=112$. As in Schrinner (2012), we also defined the local Rossby number of our simulations

$$
R_{o, l}=R_{o} \frac{\bar{\ell}}{\pi} \quad \text { where } \quad \bar{\ell}=\sum_{\ell} \ell \frac{\left\langle(\mathbf{v})_{\ell} \cdot(\mathbf{v})_{\ell}\right\rangle}{\langle\mathbf{v} \cdot \mathbf{v}\rangle}
$$

with $\mathbf{v}$ the local velocity in spherical coordinates in the frame rotating at constant angular velocity $\Omega_{0}$ and $\langle\cdot\rangle$ the average on time and radius. As seen in Figure 4 , we notice that an increasing radiative core and rotation rate lead to an increasing mean harmonic degree. In our simulations, Rossby number increases as the star ages. This result may seem counterintuitive since faster rotation rate should lead to smaller Rossby number. However, rotation rate is not the only stellar parameter changing during the PMS phase. The thickness of the convective envelop $D$ decreases at the same time. By looking at the product $\Omega_{0} D$, we see that it decreases as the star ages, leading to larger Rossby number. As the mean harmonic degree and the Rossby number grow, the local Rossby number increases as the star evolves along the PMS. By plotting $f_{\text {dip }}$ as a function of the local Rossby number, Schrinner (2012) noted a transition between the dipolar and multipolar 

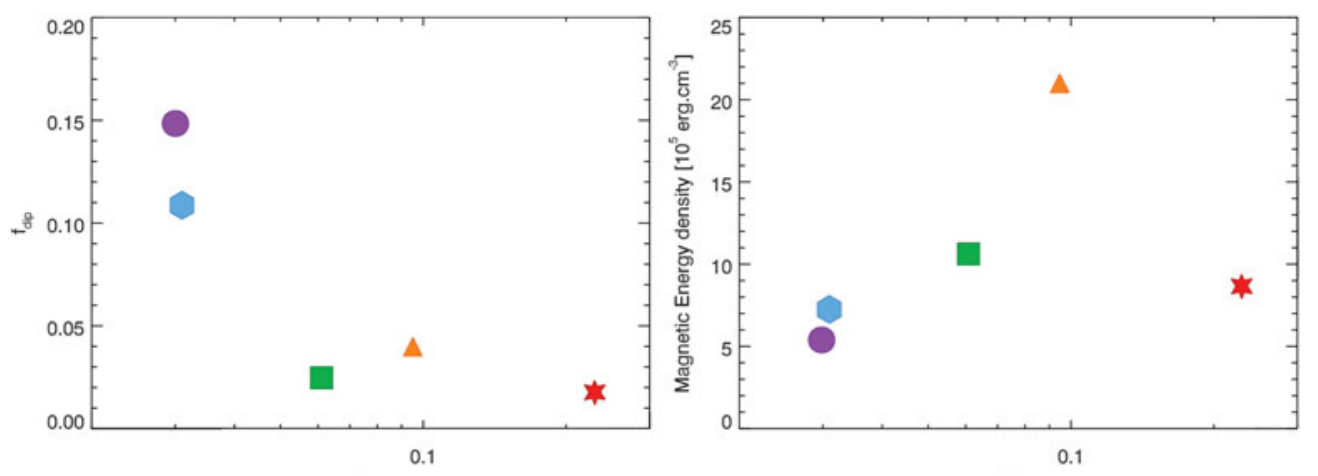

Ro,

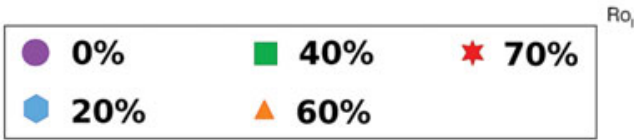

Figure 4. Evolution of the magnetic field topology with respect to the local Rossby number of our simulations. Left: Dipole field strength decreases as the local Rossby number increases. Right: Magnetic energy density evolution as the star ages along the PMS.

mode at $R_{o, l}^{c}=0.1$. The local Rossby number of our simulations are around this transition value. We also observe a transition in the topology evolution of the magnetic field along the PMS. As in Schrinner (2012), dipolar components of the magnetic field are weak when $R_{o, l}>0.1$. These components are bigger when $R_{o, l}<0.1$ even if the transition is less strong than the one observed by Schrinner. The amplitude of the magnetic field also changes as the star ages. To take into account the variation in aspect ratio of our simulations, we analyze the evolution of the magnetic energy density (ME). In figure 4, we notice that ME increases until the radiative core reaches $60 \%$ of the stellar radius and for the $70 \%$ model, ME decreases. The specificity of this simulation is a change in the stellar luminosity evolution. The size of the radiative core and the rotation rate change monotically during the PMS whereas the luminosity first decreases down to the $60 \%$ model and then increases until the ZAMS. This can explain a different behavior for the $70 \%$ model compared to the four other simulations.

For a better understanding of the dynamics of our MHD simulations, we studied the mean field generation through the analysis of the $\alpha-\Omega$ effect. We found that the influence of the $\Omega$ effect mostly increases with respect to the $\alpha$ effect. This can be understood as the $\Omega$ effect is strongly link to the differential rotation and we see that the contrast in differential rotation grows as the star evolves along the PMS. Only the $70 \%$ model behaves differently. As luminosity increases in this model, we increase both viscous and magnetic diffusivities to keep consistent Reynolds numbers. This increasing magnetic diffusivity possibly explains the behavior of the $70 \%$ model.

As the convective envelop get shallower, the radiative core grows. These radiative cores are mostly in solid rotation, i.e. do not possess differential rotation. The lack of ingredients for a dynamo generation leads us to infer, as Braithwaite (2008), that these magnetic fields in radiative zone are fossil fields, i.e. stable fields that evolves on diffusive time scales. These fields are very sensitive to instabilities and many of them are unstable and disappears quickly. These instabilities were studied by Tayler (1973) and Markey \& Tayler (1973). They showed that both purely poloidal and toroidal magnetic fields cannot be stable. Braithwaite (2008) derived a stability criteria for a stable mixed poloidal-toroidal configuration : the poloidal magnetic energy must be less that $80 \%$ of the total magnetic energy. This ratio can be rewritten as $B_{\text {pol }} / B_{\text {tor }}<2$. In Figure 5 , we 


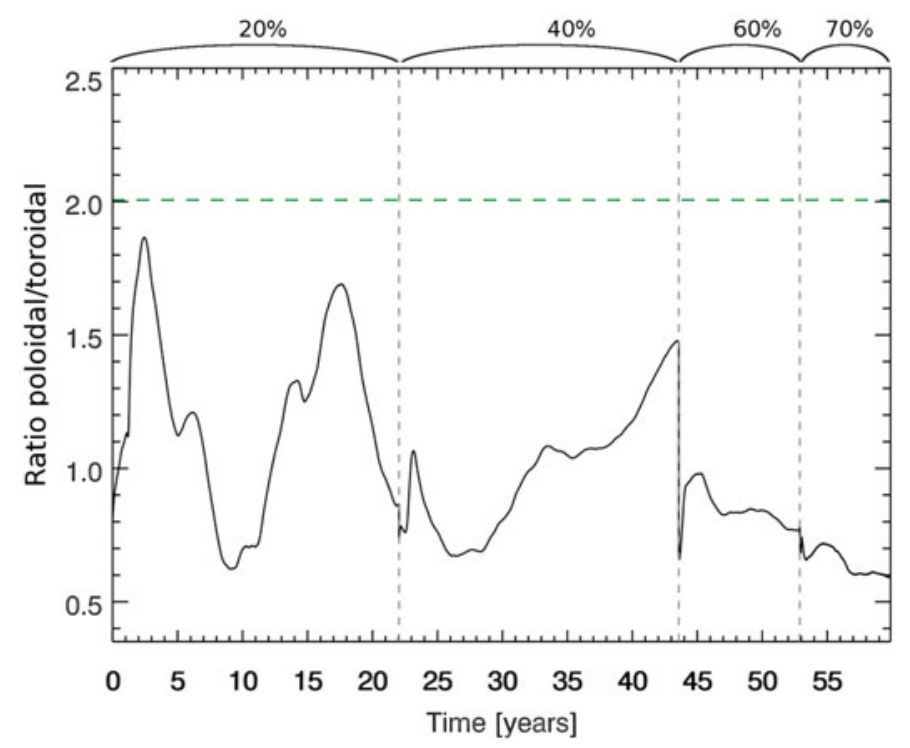

Figure 5. Evolution of the ratio $B_{\mathrm{pol}} / B_{\mathrm{tor}}$ over the PMS.

show this ratio along all our simulations. Hence we notice that the stability criteria is always fulfilled in our MHD simulations. We conclude that interestingly dynamo action tends to generate mixed fields whose properties satisfy stability criteria in stratified radiative core. This result on the stability of the fossil field left over by dynamo action as the convective envelope becomes shallower along the PMS is a direct outcome of our set of simulations and could not be easily anticipated. It has direct consequences on the geometry of fossil field that can be expected in solar-like star's radiative core as stable mixed poloidal-toroidal configurations should be favored.

\section{Conclusion}

During the PMS, between the protostellar phase and the ZAMS, the stellar radius decreases due to gravitational contraction. This stellar contraction causes an increase of the rotation rate due to angular momentum conservation. Hence we expect the star's dynamical properties to vary significantly and these variations are key to characterize. In order to do so, we have developed a series of 3D MHD simulations of stellar convective dynamo. To make this study more realistic, we used to setup the spherically symmetric background state of our 3D models, radial profiles obtained from 1D stellar evolution model at various stages along the PMS evolutionary track. We choose five different models that represent the star at specific ages of the PMS with different rotation rates and radiative radii. At first, we run hydrodynamical 3D simulations of these models in order to equilibrate internal flows and coupling between the radiative core and the convective envelop. Then we inject a magnetic field into the fully convective model and propagate it through all simulations.

Our five MHD simulations show the mutual influence of the internal magnetic field and internal flows as the star evolves along the PMS. The introduction of the magnetic field in the hydrodynamical models leads to important modifications of the internal mean flows and the convective patterns. As the differential rotation profiles are quenched by the influence of the Maxwell stresses, the radial convective patterns are larger, since they 
are less sheared. Hence viscous diffusion becomes negligible for the transport of angular momentum and inward transport is carried by Maxwell stresses.

As the star ages along the PMS, the magnetic energy in the convective envelop globally increases with a strong decrease of the mean field energy. This result is coherent with study led by Gregory et al. (2012) in which the magnetic field is less axisymmetric and more complex as the radiative core is bigger. Since we follow the evolutionary path of a solar-like star, both rotation rate and aspect ratio of the convective envelop change as the star evolves along the PMS. The specific influence of each parameter is not always easy to disentangle in our study.

The generation of the mean magnetic field shows that as the convective zone becomes shallower and the rotation rate increases, the $\Omega$ effect becomes predominant in the generation of the mean toroidal magnetic field. Moreover the $\alpha$-effect tends to generate more poloidal field than toroidal one. Hence, in each model, we see an $\alpha-\Omega$ dynamo.

As the radiative zone grows in the star, we observe that, in all models, the magnetic field in the core, left over by the convective dynamo, is stable regarding the limit given by Braithwaite (2008): $E_{\mathrm{pol}} / E_{\mathrm{tot}}<0.8$. The magnetic field in the radiative core of the star originates from the relaxation of the dynamo field coming from the previous stellar evolution phase in our sequence of models. By looking at this dynamo field, we notice, that in all convection zones, the magnetic field that comes from the dynamo action also fulfill the stability criteria $E_{\mathrm{pol}} / E_{\mathrm{tot}}<0.8$ even if this has no obvious consequence in that zone.

The global properties of the magnetic fields we obtain in our study also have direct consequences on the coronae of PMS stars. We have computed the change of the Alfvén radius (e.g. the radius where the stellar wind decouples from the star) that such topological and rotation state implies, following the prescription described in Réville (2015). We find that the Alfvén radius shrink from about $33 R_{\odot}$ to $10 R_{\odot}$. This is in good qualitative agreement with the recent work of Réville (2016) who computed realistic 3D stellar wind along the evolutionary track of a solar mass star using spectro-polarimetric maps from Folsom (2016). We intend in the near future to use the magnetic field coming out of our dynamo simulations to compute similar 3D wind solutions along the PMS. This will allow us to assess the loss of mass and angular momentum, that must also vary significantly given the large change of Alfvén radius we have identified. It is important to notice that in this study, we choose our models to follow an astrophysical path along the PMS. A logical follow-up is therefore to apply this analysis to the evolution of solar-like stars along the following step of stellar evolution, i.e. the main sequence. In that study the main parameter will be the decrease of the rotation rate as the star is braked by the solar wind and the internal stellar structure of the star remains almost unchanged during this evolutionary phase. An additional study would be to study the impact of stellar structure with a fixed rotation rate. We have started doing such studies and their results will be reported in future communications.

\section{References}

Amard, L., Palacios, A., Charbonnel, C., Gallet, F. \& Bouvier, J. 2016 A\&\&A, 587, A105

Braithwaite, J. 2008 MNRAS, 386, 1947-1958

Brun, A. S., Miesch, M. S., \& Toomre, J. 2004, ApJ, 614, 1073

Christensen, U. R. \& Aubert, J. 2006, Geophysical Journal International, 166, 97-114

Emeriau-Viard, C. \& Brun, A. S. 2017 ApJ, submitted

Folsom, C. P., Petit, P. , Bouvier, J., Lèbre, A., Amard, L., Palacios, A., Morin, J., Donati, J.-F., Jeffers, S. V., Marsden, S. C. \& Vidotto, A. A. 2016 MNRAS, 457, 580-607 
Gallet, F. \& Bouvier, J. 2013, A\&A, 556, A36

Gregory, S. G. Donati, J.-F., Morin, J., Hussain, G. A. J., Mayne, N. J., Hillenbrand, L. A. \& Jardine, M. 2012 ApJ, 755, 97

MacGregor, K. B. \& Brenner, M. 1991, ApJ, 376, 204-213

Markey, P. \& Tayler, R. J. 1973 MNRAS, 163, 77

Morin, J., Donati, J.-F., Petit, P., Delfosse, X., Forveille, T. \& Jardine, M. M., 2010 MNRAS, 407, 2269-2286

Pizzolato, N., Maggio, A., Micela, G., Sciortino, S. \& Ventura, 2003 A\&A, 397, 147-157

Réville, V., Brun, A. S., Matt, S. P., Strugarek, A. \& Pinto, R. F. 2015 ApJ, 798, 116

Réville, V., Brun, A. S., Strugarek, A., Matt, S. P., Bouvier, J., Folsom, C. P., \& Petit, P. 2015, ApJ, 814, 99

Réville, V., Folsom, C. P., Strugarek, A.\& Brun, A. S. 2016 ApJ, 832, 145

Schrinner, M., Petitdemange, L. \& Dormy, E. 2012 ApJ, 752, 121

Strugarek, A., Beaudoin, P., Charbonneau, P., Brun, A.S. \& do Nascimento, J.-D. 2017 submitted

Tayler, R. J. 1973 MNRAS, 161, 365 